\title{
Resistance to extinction as a function of level of discriminative training and the presence or absence of an irrelevant cue
}

\section{B. R. HERGENHAHN ${ }^{1}$ AND JACK CAPEHART}

UNIVERSITY OF ARIZONA

The hypothesis that behavior becomes increasingly independent of irrelevant cues as a result of overtraining was tested by partially replicating a study of D'Amato, Schiff, \&+ Jagoda (1962). The hypothesis was not confimed; behavior was found to become more under the influence of irrelevant cues with prolonged training as measured by resistance to extinction.

D'Amato, Schiff, \& Jagoda (1962) tested and confirmed the hypothesis that, with albino rats, prolonged training reduces resistance to extinction for a discriminative operant response but not for an instrumental response. This hypothesis was based on the assumption that Ss being trained on a discriminative task are on a partial reinforcement schedule until the task is solved, at which point continuous reinforcement ensues. Accordingly, overtraining removes $S$ from a partial reinforcement situation and exposes him, essentially, to $100 \%$ reinforcement. Because extinction is more rapid following $100 \%$ reinforcement than it is following reinforcement at a percentage less than $100 \%$, overtrained Ss extinguish more rapidly than criterion trained Ss. Where simple nondiscriminative training is involved, reduction in resistance to extinction does not take place because reinforcement is continuous from the start. Resistance to extinction following both discriminative and nondiscriminative training was measured after $200,400,800$, or 1600 reinforcements.

It seems possible that these results could be explained in another way. Irrelevant cues present during discrimination training could become adapted in a manner suggested by Restle (1955) and thereby have relatively less influence on the behavior of overtrained Ss during extinction. In other words, overtrained Ss would not have to extinguish responses to as many cues as criterion trained Ss and would therefore extinguish faster.

The click of the feeder mechanism would be an irrelevant cue if it resulted from a bar press both during the $\mathrm{S}^{\mathrm{D}}$ and the $\mathrm{S}^{\Delta}$ periods. In the $\mathrm{D}^{\prime}$ Amato et al study (1962) the feeder mechanism was disconnected during the $S^{\Delta}$ periods (personal communication from D'Amato, 1965) and therefore the click could not have functioned as an irrelevant cue. However, it is assumed that many other irrelevant cues were present; in fact, any stimulus that was present both during the $\mathrm{S}^{\mathrm{D}}$ and
$\mathrm{S}^{\Delta}$ periods would be an example. Of course such cues would only be involved in discrimination training; for Ss receiving simple instrumental training all cues are presumably relevant. It is important to note that having the click of the feeder mechanism occur only during the $\mathrm{S}^{\mathrm{D}}$ period made it, in a sense, a second relevant cue. Both the cue 1ight and the click were perfect predictors of reward, and under these conditions, it seems possible that Ss could have solved the discrimination problem without utilizing the light at all or by using a combination of the cue light and the click.

To investigate the importance of the adaptation of irrelevant cues the D'Amato et al study (1962) was partially replicated. Training levels of 400 and 1600 reinforcements were employed. The click of the feeder mechanism resulted from a bar press during both the $\mathrm{S}^{D}$ and $\mathrm{s}^{\Delta}$ periods, making it irrelevant. For one-half Ss receiving 400 reinforcements the click of the feeder mechanism resulted from a bar press during extinction and for the other one-half there was no click. The same was true for Ss receiving 1600 reinforcements during acquisition. There were five Ss in each of the four extinction conditions. All other aspects of the experiment were equivalent to those reported by D'Amato et al (1962).

A significant interaction between level of training (400 vs. 1600) and click vs. no click during extinction was obtained $(F=10.39, \mathrm{df}=1 / 16, p<.01)$. Ss without the click present extinguished more slowly after 400 acquisition trials and faster after 1600 trials. The reverse was true for the groups who experienced the click during extinction. This interaction is shown in Fig. 1.

Within the limits of training used, these results seem to indicate that behavior did not become increasingly independent of irrelevant cues following prolonged training. In fact the opposite was the case and this is precisely what reinforcement theory would have predicted. That is, as training level increased, the click became a more powerful "secondary reinforcer" and, therefore, its presence prolonged extinction. It is possible, however, that inserting the irrelevant cue into the problem made the discrimination more difficult. Almost certainly the problem was more difficult than the one employed by D'Amato et al (1962) in which there were two relevant cues. Therefore, 


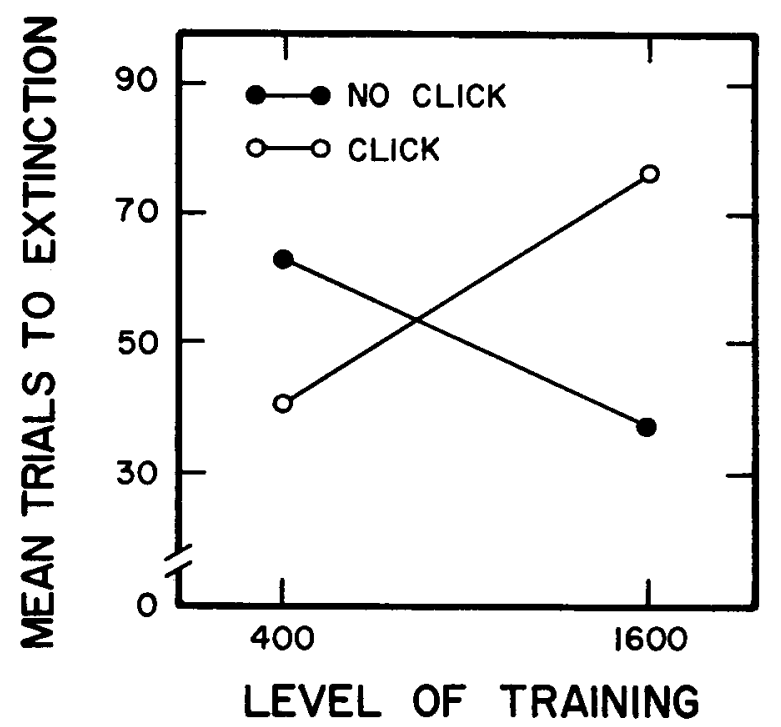

Fig. 1. Mean trials to extinction as a function of training level and the presence or absence of the feeder mechanism click.

it may be that Ss receiving 1600 reinforcements in the present study were more equivalent to Ss receiving 800 reinforcements in the D'Amato et al study. Trials that were sufficient to test the hypothesis of D'Amato et al may not have been enough to determine whether or not an irrelevant cue had been adapted in the present study. If this was the case, it points up a very important difference between overtraining and overlearning. It is always possible, where a fixed number of trials is involved, that the former is taking place but not necessarily the latter. As a result it still seems possible that reduced resistance to extinction following prolonged discriminative training may be partially explained by an adaptation process of the kind suggested by Restle (1955). Any attempt to evaluate this process, however, must take into consideration the increased difficulty resulting from the insertion of irrelevant cues into the problem.

\section{References}

D'Amato, M. R. Personal Communication, 1965.

D'Amato, M. R., Schiff, D., \& Jagoda, H. Resistance to extinction after varying amounts of discriminative or nondiscriminative instrumental training. J. exp. Psychol., 1962, 64, 520-532.

Restle, F. A. A theory of discrimination learning. Psychol. Rev., $1955,62,11-19$.

\section{Note}

1. Now at Hamine University. 